\begin{tabular}{|c|l|}
\hline Title & $\begin{array}{l}\text { Ferromagnetic nanoclusters hy bridized in Mn-incorporated Gal nA s layers during metal-organic vapour phase epitaxial } \\
\text { growth on InP lay ers under low growth temperature conditions }\end{array}$ \\
\hline Author(s) & Hara, Shinjiroh; Kuramata, A kito \\
\hline Citation & $\begin{array}{l}\text { Nanotechnology, 16/6), 957-965 } \\
\text { https://doi.org/10.1088/0957-4484/16/6/057 }\end{array}$ \\
\hline Issue Date & 2005-06 \\
\hline Doc URL & http://hdl.handle.net/2115/5870 \\
\hline Rights & Copyright $\odot$ 2005 IOP Publishing Ltd. \\
\hline Type & article (author version) \\
\hline Note(URL) & Nttp://www.iop.org/ \\
\hline Information & Nanotech16-6.pdf \\
\hline
\end{tabular}

Instructions for use 


\title{
Ferromagnetic nanoclusters hybridized in Mn-incorporated GaInAs layers during metal-organic vapour phase epitaxial growth on InP layers under low growth temperature conditions
}

\author{
Shinjiroh Hara * and Akito Kuramata \\ Fujitsu Laboratories Ltd., 10-1 Morinosato-Wakamiya, Atsugi 243-0197, Japan \\ E-mai: hara@rciqe.hokudai.ac.jp
}

Received 3 November 2004, in final form 11 March 2005

Published 21 April 2005

Online at stacks.iop.org/Nano/16/957

\begin{abstract}
We demonstrate the successful formation of ferromagnetic nanoclusters in Mn-incorporated GaInAs layers grown by metal-organic vapour phase epitaxy on InP (100) substrates under low growth temperature conditions below $450{ }^{\circ} \mathrm{C}$. We find that MnAs nanoclusters with NiAs-type hexagonal crystallographic structures, which show ferromagnetic characteristics up to a relatively high temperature of about $305 \mathrm{~K}$, are formed near the layer surfaces of Mn-incorporated GaInAs layers grown at $440{ }^{\circ} \mathrm{C}$. After deposition of undoped InP layers on Mn-incorporated GaInAs layers, MnP nanoclusters with orthorhombic cubic crystallographic structures, in which $7 \%$ arsenic is incorporated, are formed in InP layers. The samples with $\mathrm{MnP}$ nanoclusters show strong ferromagnetic coupling up to about $305 \mathrm{~K}$, although the Curie temperature of $\mathrm{MnP}$ bulk compounds is $291 \mathrm{~K}$. Energy dispersive $\mathrm{x}$-ray spectroscopy (EDS) indicates that Mn concentrations in InP and GaInAs layers surrounding $\mathrm{MnP}$ nanoclusters are almost negligible. MnAs nanoclusters are also formed in Mn-incorporated GaAs layers grown under low growth temperature conditions of $450{ }^{\circ} \mathrm{C}$ on GaAs (100) substrates. From the results of magnetic characterizations with respect to growth temperatures of the samples, we found that all the Mn-incorporated GaAs layers grown at temperatures below $450{ }^{\circ} \mathrm{C}$ showed ferromagnetic behaviour.
\end{abstract}

PACS: 61.46.+w, 68.37.Lp, 68.65.-k, 75.75.+a, 81.07.-b, 81.10.-h, 81.15.Gh, 81.16.-c

Keywords

Ferromagnetism, nanoclusters, MOVPE, GaInAs, InP, GaAs, MnAs, MnP

* Author to whom any correspondence should be addressed.

Present address: Graduate School of Information Science and Technology, Hokkaido University, North 14 West 9, Sapporo 060-0814, Japan.

Fax: +81-11-716-6004, Phone: +81-11-706-7176 


\section{Introduction}

III-V compound semiconductor-based diluted magnetic semiconductor (III-V DMS) alloys and ferromagnetic nanoclusters hybridized in III-V compound semiconductors (FM III-V hybrids) have attracted much interest in recent years because of their enormous potential realizing new electronic devices in the next generation using not only the charge but also the spin of electrons. The III-V DMS materials and FM III-V hybrids investigated most intensively were GaAs-related materials, such as GaAs:MnAs [1], GaMnAs [2], InMnAs [2, 3] and so on (for a review, see [1, 2]). Almost all of these materials have been grown by low temperature molecular beam epitaxy (LTMBE) or ion implantation of magnetic materials on semiconductors [4]. However, although metal-organic vapour phase epitaxy (MOVPE) is one of the most important and preferred technologies for the fabrication of electronic and photonic semiconductor devices, particularly in a production fashion, there are nevertheless only a few reports on MOVPE growth of III-V DMS materials and FM III-V hybrids [5 - 8].

Magneto-optical devices utilizing the Faraday or Kerr effect for III-V DMS materials or FM III-V hybrids are very promising for realizing monolithic integration of magneto-optical devices such as waveguide-type optical isolators or circulators on the present III-V compound semiconductor-based photonic integrated circuits (PICs). This is because such a material system is compatible with III-V compound semiconductors such as GaAs and InP. In particular, FM III-V hybrids are more suitable because they show strong ferromagnetic coupling even above room temperature. Optical isolators are key devices in optical communication systems for 1.3 or $1.55 \mu \mathrm{m}$ wavelength bands for reducing the external relative feedback noise in distributed feedback (DFB) lasers integrated on the PICs. Techniques for achieving direct bonding between semiconductors and magneto-optical materials have been proposed [9]. However, no successful monolithic integration of optical isolators using epitaxial growth techniques has been realized so far for the present III-V compound semiconductor-based PICs. Recently, device structures for waveguide-type optical isolators using MnAs nanoclusters grown by LT-MBE on GaAs substrates have been proposed and demonstrated [10]. In order to fabricate PICs such as highly integrated dense wavelength division multiplexing (DWDM) devices for optical communication systems, InP-related materials, e.g. GaInAs, GaInAsP and AlGaInAs, have been commonly and preferentially used and will be used for years to come. Several investigations of GaInMnAs DMS alloys grown by LT-MBE have been reported as the first results based on InP-related materials in recent years [11, 12]. However, until now, no work has focused on MOVPE growth of magnetic materials hybridized in InP-related materials. In our previous studies, the first demonstration of MnAs nanocluster formation in Mn-incorporated GaInAs layers on InP layers grown by MOVPE has been reported [8]. Using tertiary-butyl-arsine (TBAs) as a group V source material for MOVPE growth, MnAs nanoclusters were formed in Mn-incorporated GaInAs layers grown at relatively high growth temperatures of 500 and $590{ }^{\circ} \mathrm{C}$; on the other hand, under low growth temperature conditions below $450{ }^{\circ} \mathrm{C}$, whisker structures were formed [8]. It is quite important to understand MOVPE growth of binary and/or ternary semiconductor compounds combined with ferromagnetic materials because it leads to realization of waveguide-type optical isolators using ferromagnetic materials hybridized in quaternary semiconductor compounds such as GaInAsP and $\mathrm{AlGaInAs,}$ which are preferentially used in optical communication systems.

In this paper, we demonstrate ferromagnetic MnAs nanocluster formation in Mn-incorporated GaInAs layers grown even at low growth temperatures below $450{ }^{\circ} \mathrm{C}$ by MOVPE using arsine $\left(\mathrm{AsH}_{3}\right)$ as a group V source material. From the view point of realistic device applications of FM III-V hybrids, what we need is to realize heterostructures from compound semiconductors, e.g. GaInAs, GaInAsP, InP and AlGaInAs, combined with ferromagnetic materials. Therefore, this paper also describes fundamental growth studies and magnetic characterizations of ferromagnetic nanoclusters formed in heterostructures between undoped $\mathrm{InP}$ and $\mathrm{Mn}$-incorporated GaInAs layers. The results indicate that ferromagnetic MnP nanoclusters are formed in the heterostructures.

\section{Experimental procedures}

A vertical low pressure MOVPE system was used with triethyl gallium (TEGa) and trimethyl indium (TMIn) as group III source materials, and with conventional hydride precursors like phosphine $\left(\mathrm{PH}_{3}\right)$ and arsine $\left(\mathrm{AsH}_{3}\right)$ as group $\mathrm{V}$ source materials. The working reactor pressure was maintained at 50 Torr. Bis-(methyl-cyclopentadienyl) manganese $\left((\mathrm{MeCp})_{2} \mathrm{Mn}\right)$ was used as an organometallic source material for manganese. Figure 1 shows a schematic cartoon of the supply system set-up for $(\mathrm{MeCp})_{2} \mathrm{Mn}$ in our MOVPE system. $(\mathrm{MeCp})_{2} \mathrm{Mn}$ is in the solid phase at room temperature and has a very low vapour pressure, 
e.g. the estimated vapour pressure of $(\mathrm{MeCp})_{2} \mathrm{Mn}$ at $37{ }^{\circ} \mathrm{C}$ is only around 0.016 Torr. Its melting point is $62{ }^{\circ} \mathrm{C}$. In order to efficiently introduce $(\mathrm{MeCp})_{2} \mathrm{Mn}$ to semiconductor wafer surfaces in the MOVPE reactor, the temperature of $(\mathrm{MeCp})_{2} \mathrm{Mn}$ and its gas inlet to the reactor were heated to 70 and $75^{\circ} \mathrm{C}$, respectively. The estimated vapour pressure of $(\mathrm{MeCp})_{2} \mathrm{Mn}$ at $70{ }^{\circ} \mathrm{C}$ is around 0.25 Torr.

The growth temperatures, $\mathrm{T}_{\mathrm{g}}\left({ }^{\circ} \mathrm{C}\right)$, were varied from 380 to $450{ }^{\circ} \mathrm{C}$ for Mn-incorporated GaInAs layers. Here, the growth temperatures, $\mathrm{T}_{\mathrm{g}}$, referred to in the article are the temperatures measured by thermocouples inside carbon trays for setting semiconductor wafers on. The actual temperature of the wafer surface during growth can be higher than $\mathrm{T}_{\mathrm{g}}$ by around $10^{\circ} \mathrm{C}$ at most, as a result of temperature calibration. Typical layer structures are schematically depicted in figure 2 . In order to fundamentally study growth morphologies and magnetic properties of magnetic nanoclusters in heterostructures, 'TYPE-I' (without undoped InP cap layers) and 'TYPE-II' (with undoped InP cap layers) layer sequences were chosen in this study. The TYPE-I layer sequence was as follows: Mn-incorporated GaInAs / undoped GaInAs / undoped InP buffer layers. The other structure, the TYPE-II layer sequence, was made of undoped InP cap / Mn-incorporated GaInAs / undoped InP buffer layers. Both layer structures were grown on InP (100) n-type substrates. $(\mathrm{MeCp})_{2} \mathrm{Mn}$ was always introduced during the growth of GaInAs layers. However, InP cap and buffer layers were always undoped. The nominal $\mathrm{V} /(\mathrm{III}+\mathrm{Mn})$ ratio in the vapour phase for Mn-incorporated GaInAs layers was 250 and the nominal V/III ratio for InP cap layers was 400 at low growth temperatures below $450{ }^{\circ} \mathrm{C}$. It should be noted that thermal decomposition rates of precursors become lower with decreasing growth temperature in the low growth temperature regime used in this study. Typical supply gas ratios in the vapour phase between the total partial pressures of group III source materials and those of $(\mathrm{MeCp})_{2} \mathrm{Mn}$, that is, $x=\mathrm{Mn} /(\mathrm{III}+\mathrm{Mn})$, were changed from 0.02 to 0.06 . Here, the supply gas ratios of partial pressure of $(\mathrm{MeCp})_{2} \mathrm{Mn}$ to the total one of group III source materials and $(\mathrm{MeCp})_{2} \mathrm{Mn}, x$, that is, $x=$ $\mathrm{p}\left[(\mathrm{MeCp})_{2} \mathrm{Mn}\right] /\left(\mathrm{p}\right.$ [group III sources] $\left.+\mathrm{p}\left[(\mathrm{MeCp})_{2} \mathrm{Mn}\right]\right)$, are referred to as ' $\mathrm{Mn} /(\mathrm{III}+\mathrm{Mn})$ ratios in the vapour phase', $x$, in this paper. The growth rates of GaInAs layers almost lattice matched on InP layers at 400 and $450{ }^{\circ} \mathrm{C}$ were about 0.72 and $1.0 \AA \mathrm{s}^{-1}$, respectively. Growth temperatures for undoped InP cap layers of TYPE-II layer structures were the same as the ones, which were below $450{ }^{\circ} \mathrm{C}$, for $\mathrm{Mn}$-incorporated GaInAs layers during the same growth runs. Only for undoped $\mathrm{InP}$ buffer layers directly grown on $\mathrm{InP}(100)$ substrates were conventional MOVPE growth conditions, that is, $\mathrm{T}_{\mathrm{g}}=630^{\circ} \mathrm{C}$ and $\mathrm{V} / \mathrm{III}$ $=100$, chosen. To grow Mn-incorporated GaInAs layers at $380-450{ }^{\circ} \mathrm{C}$, growth interruptions for 7 min were introduced after the growth of undoped InP buffer layers grown at $630{ }^{\circ} \mathrm{C}$. In order to prevent unintentional manganese incorporation into the epitaxial layers, carbon trays for setting wafers on were heated in a hydrogen atmosphere above $700{ }^{\circ} \mathrm{C}$ for more than $30 \mathrm{~min}$ after each of the growth runs for $\mathrm{Mn}$-incorporated samples. Comparing two samples, which were grown under the same growth conditions, before and after such a process of heating of the carbon trays, we observed no changes in x-ray diffraction (XRD) spectra from Mn-incorporated layers of the two samples grown at $400{ }^{\circ} \mathrm{C}$ on buffer layers grown at $650^{\circ} \mathrm{C}$.

Cross-sectional images of the samples were observed by transmission electron microscopy (TEM). Energy dispersive x-ray spectroscopy (EDS) using an electron beam with a spot diameter of about $1 \mathrm{~nm}$ was carried out for compositional characterizations of layers and nanoclusters. Electron beam diffraction patterns were taken to identify crystallographic structures of nanoclusters embedded in host semiconductor layers. Magnetic properties (M-H curves) of TYPE-I and TYPE-II layer structures were characterized using superconducting quantum interference device (SQUID) magnetometers. We measured the M-H curves at different temperatures around room temperature to examine the magnetic characteristics of the samples. External magnetic fields, $\mathbf{H}$, were applied in a direction parallel to the substrates plane, $\mathbf{H} / /[011]$ in this study. From a rough estimation, the samples for all of the SQUID characterizations measured about $0.4 \mathrm{~cm}$ long, $0.3-0.35 \mathrm{~cm}$ wide and $350 \mu \mathrm{m}$ thick (almost same as the substrates thickness). However, all the magnetization values for $\mathrm{M}-\mathrm{H}$ curves in this article were not normalized by the volumes of the samples.

\section{Structural characterizations of nanoclusters}

Figure 3(a) shows structural characterization results obtained by cross-sectional TEM observations and electron beam diffraction patterns for TYPE-I layer structures grown at $440{ }^{\circ} \mathrm{C}$. It was clearly observed that several nanoclusters were formed near the surfaces of Mn-incorporated GaInAs layers (indicated by small white arrows in the top image), as shown in the TEM image. The typical nanoclusters measured $55-70 \mathrm{~nm}$ wide and $25-40 \mathrm{~nm}$ high from the cross-sectional observations. The white rectangular marker in Figure 3(a) represents a typical observation area for taking a highly magnified image. Using electron beam 
diffraction from [1-1-1] injection for the nanoclusters, we found that these nanoclusters had NiAs-type hexagonal crystallographic structures with lattice constants of $\mathrm{a}=0.372 \mathrm{~nm}$ and $\mathrm{c}=0.571 \mathrm{~nm}$, that is, MnAs. These results were supported by compositional analysis using EDS, as shown in Table 1. The averaged atomic concentrations (\%) of five elements, In, Ga, As, P, and Mn, are summarized in table 1. The nanoclusters in TYPE-I layer structures consisted of a mixture of manganese and arsenic atoms, whereas the $\mathrm{Mn}$ concentrations in the surrounding GaInAs layers were almost negligible. As shown in a highly magnified lattice image taken with [011] projection for zinc-blende GaInAs layers surrounding the nanocluster, we found that (101) planes of the MnAs nanoclusters were oriented nearly parallel to (111) planes of the surrounding GaInAs layers. The relationship of the crystallographic orientations of the MnAs nanoclusters and GaInAs layers was roughly estimated. An electron beam diffraction pattern from the MnAs nanoclusters in the middle in figure 3(a) was taken from the [011] injection for the surrounding GaInAs layers, and that at the bottom was taken by tilting the samples for the MnAs nanoclusters. The results indicated that the [1-1-1] direction of the MnAs nanoclusters was tilted by around $4^{\circ}$ toward a [-11-2] direction. For TYPE-I layer structures grown at $380{ }^{\circ} \mathrm{C}$, on the other hand, cross-sectional TEM observations showed that no MnAs nanoclusters were formed in Mn-incorporated GaInAs layers. In addition, EDS analysis indicated that Mn concentrations in the Mn-incorporated GaInAs layers were almost negligible, less than $1 \%$. We concluded from electron beam diffraction patterns that Mn-incorporated GaInAs layers of the TYPE-I layer structures at $380{ }^{\circ} \mathrm{C}$ were epitaxially grown on undoped GaInAs/InP (100) layers, although the Mn-incorporated layers seemed not to be completely homogeneous from the results of cross-sectional TEM observations (not shown here).

Next, structural characterizations were carried out for TYPE-II layer structures. Figure 3(b) shows cross-sectional TEM images of the observed heterostructure with 'trilayers' of undoped InP and Mn-incorporated GaInAs layers and electron beam diffraction patterns from InP, Mn-incorporated GaInAs layers and nanoclusters. (MeCp) ${ }_{2} \mathrm{Mn}$ was introduced during the growth of GaInAs layers like in the case of TYPE-I layer structures in figure 3(a). The growth temperature was $450{ }^{\circ} \mathrm{C}$. No nanoclusters were formed in $\mathrm{Mn}$-incorporated GaInAs layers but they were formed in InP spacer and cap layers (indicated by small white arrows in the top image). A highly magnified lattice image of the nanoclusters observed in the InP layers was taken with [0-11] projection for zinc-blende InP layers surrounding the nanoclusters. The white rectangular marker in figure 3(b) represents a typical observation area for taking a highly magnified image. Clear lattice images were observed for the surrounding InP layers. In contrast, the areas of nanoclusters showed abstract views. However, we obtained clear electron beam diffraction patterns from these nanoclusters whose crystallographic planes were tilted by about $10^{\circ}$ to the surrounding zinc-blende-type InP and GaInAs crystallographic planes, as shown in figure 3(b). The relationship of the crystallographic directions between the nanoclusters and the surrounding zinc-blende layers has not been determined precisely yet in this study. However, we concluded that these nanoclusters had MnP-type orthorhombic crystallographic structures $(P c m n: \mathrm{a}=0.591 \mathrm{~nm}, \mathrm{~b}=0.525 \mathrm{~nm}, \mathrm{c}=0.317 \mathrm{~nm})$ from the results of electron beam diffractions.

As shown in the EDS analysis results summarized in Table 1, the MnP-type orthorhombic nanoclusters embedded in the InP layers observed in the TEM images in figure 3(b) consisted of a mixture of manganese and phosphorus atoms in solid composition. However, about $7 \%$ arsenic atoms were also contained in the nanoclusters. Though $(\mathrm{MeCp})_{2} \mathrm{Mn}$ was always introduced during the growth of GaInAs layers, Mn concentrations in the GaInAs layers were nevertheless almost negligible. Therefore, it is presumably possible that $\mathrm{Mn}$ atoms initially incorporated in GaInAs layers were segregated to the GaInAs layer surfaces during the growth, and then such surface-segregated Mn atoms (or MnAs grains) near the GaInAs layer surfaces reacted with phosphorus precursors during the subsequent growth of InP cap layers. Eventually, MnP nanoclusters were formed in InP layers. Thermodynamically speaking, MnP compounds can be more stable than MnAs ones. The standard enthalpies of formation reactions of the elements, $\Delta \mathrm{H}_{\mathrm{f}}$ $\left(298 \mathrm{~K}\right.$ ), for MnAs and MnP binary compounds are -56.90 and $-112.97 \mathrm{~kJ} \mathrm{~mol}^{-1}$, respectively, and their $\Delta \mathrm{H}_{\mathrm{f}}$ values at $700 \mathrm{~K}$ (at about $430{ }^{\circ} \mathrm{C}$ ) are -47.09 and $-114.73 \mathrm{~kJ} \mathrm{~mol}^{-1}$, respectively [13]. Therefore, even though surface-segregated $\mathrm{Mn}$ atoms formed $\mathrm{Mn}$-As bonds near the GaInAs layer surfaces at the growth temperature of $450^{\circ} \mathrm{C}, \mathrm{MnP}$ compounds should be formed during the following InP layer growth.

\section{Magnetic properties of nanoclusters}

Initially, in order to investigate magnetic properties of the nanoclusters formed in the Mn-incorporated GaInAs layers, SQUID measurements were carried out for the TYPE-I layer structures shown in figure 3(a). 
Figures 4(a) and (b) show the M-H curves at various temperatures for the samples grown at 440 and $380{ }^{\circ} \mathrm{C}$, respectively. The $\mathrm{Mn} /(\mathrm{III}+\mathrm{Mn})$ ratio in the vapour phase, $x$, was 0.04 for both samples. A reference sample was made of undoped GaInAs and undoped InP layers grown on InP (100) substrates. In all of the M-H curves shown in figure 4, diamagnetic characteristics from GaInAs and $\mathrm{InP}$ layers of the reference samples were subtracted. For the samples grown at $440{ }^{\circ} \mathrm{C}$, ferromagnetic characteristics (or hysteresis loops in $\mathrm{M}-\mathrm{H}$ curves) were observed up to about $305 \mathrm{~K}$, and the samples showed paramagnetic behaviours at $330 \mathrm{~K}$, as shown in figure 4(a). On the other hand, for the samples grown at $380{ }^{\circ} \mathrm{C}$, the ferromagnetic characteristics were retained up to about $130 \mathrm{~K}$. However, clear hysteresis loops were not observed at 270 $\mathrm{K}$ and the saturation magnetization was also decreased, although the samples still showed ferromagnetic coupling, as shown in figure 4(b). GaInMnAs DMS alloy layers grown by LT-MBE have been reported to have a Curie temperature of about $100 \mathrm{~K}$ or less, in which magnetic fields applied for the SQUID measurements were in a direction parallel to the substrates plane in [11] and perpendicular to it, $\mathbf{H} / /$ [100], in [12]. It was reported there that the magnetization easy axis was in the plane of the layers in [11] and perpendicular to the plane in [12]. In this study, magnetic fields were applied in a direction parallel to the substrate plane, $\mathbf{H} / /$ [011]. At present, it is not clear whether GaInMnAs DMS alloy layers were formed in the TYPE-I layer structures grown at $380{ }^{\circ} \mathrm{C}$ by MOVPE in this study, and whether the difference in observed magnetic behaviours shown in figure 4 may indicate magnetic anisotropy of the layers and/or nanoclusters, that is, a difference in magnetization easy axis between the samples grown at 440 and $380{ }^{\circ} \mathrm{C}$. However, the present results obtained in the magnetic characterizations seem to be consistent with the structural characterization results shown in the previous section, in which we showed MnAs nanocluster formation for the samples grown at $440{ }^{\circ} \mathrm{C}$ and no cluster formation for the ones grown at $380{ }^{\circ} \mathrm{C}$. We are currently performing further structural characterizations of the samples and MOVPE growth experiments to clarify growth condition dependence of the morphologies of Mn-incorporated GaInAs layers in detail.

Subsequently, SQUID measurements were carried out for the samples with TYPE-II layer structures. Figure 5 shows M-H curves for the TYPE-II layer structures with the MnP nanoclusters (solid lines) and a reference sample with undoped GaInAs and undoped InP layers (dashed lines) measured by SQUID magnetometers. For all of the measurements, magnetic fields, H, were applied in a [011] direction. The results indicate that diamagnetic characteristics from GaInAs and InP layers are added to the ferromagnetic behaviours of the TYPE-II layer structures. Figure 5(a) shows the dependence of the M-H curves on the growth temperatures, $\mathrm{T}_{\mathrm{g}}\left({ }^{\circ} \mathrm{C}\right)$, of the samples. The lower the growth temperatures the samples were grown at, the larger the saturation magnetization, $\mathbf{M}_{\mathbf{s}}$, values were. This is presumably because of the higher rates of re-evaporation of $\mathrm{Mn}$ atoms from the layers grown at $450{ }^{\circ} \mathrm{C}$ compared with those at $400{ }^{\circ} \mathrm{C}$. However, other manganese-phosphorus compounds such as $\mathrm{Mn}_{2} \mathrm{P}$, which shows antiferromagnetic ordering and has a crystallographic structure of a revised hexagonal $\mathrm{Fe}_{2} \mathrm{P}$-type $(P-62 \mathrm{~m}: \mathrm{a}=0.608 \mathrm{~nm}, \mathrm{c}=0.346 \mathrm{~nm})$ [14], are also possibly formed in the layers. In the TYPE-II layers grown at $450{ }^{\circ} \mathrm{C}$, atomic concentrations in the clusters summarized in Table 1 and the electron diffraction pattern in figure 3(b) supported the suggestion of formation of $\mathrm{MnP}$ nanoclusters in the layers. We believe that ferromagnetic MnP nanoclusters were probably formed in the samples grown at $400{ }^{\circ} \mathrm{C}$ because the magnetic behaviours were similar to those in the case of the TYPE-II layers grown at $450{ }^{\circ} \mathrm{C}$. To clarify the situation for the layers grown at $400{ }^{\circ} \mathrm{C}$, however, we still need further structural analysis of the nanoclusters, in detail. The saturation magnetization values were also increased by increasing the $\mathrm{Mn} /(\mathrm{III}+\mathrm{Mn})$ ratio in the vapour phase, $x$, from 0.04 to 0.06 , as shown in figure 5(b). The growth temperatures for the samples with MnP nanoclusters in figure 5(b) were $400{ }^{\circ} \mathrm{C}$. The results, therefore, indicate that manganese incorporations into epitaxial layers can be controlled by changing the growth temperatures and partial pressures of $(\mathrm{MeCp})_{2} \mathrm{Mn}$ in MOVPE growth. Here, it should be noted that the differences in saturation magnetization values in figures 5(a) and (b) involve some amount of influence of the differences of sample size.

Figure 5(c) shows the M-H curves at various temperatures for the samples with $\mathrm{MnP}$ nanoclusters grown at $400{ }^{\circ} \mathrm{C}$. The $\mathrm{Mn} /(\mathrm{III}+\mathrm{Mn})$ ratio in the vapour phase, $x$, was set to 0.04 in figure $5(\mathrm{c})$. We found that the TYPE-II layer structures with MnP nanoclusters showed much larger ferromagnetic coupling than the TYPE-I layer structures with MnAs nanoclusters. Clear hysteresis loops were observed up to $305 \mathrm{~K}$. The Curie temperature, $\mathrm{T}_{\mathfrak{c}}$, of single-crystal $\mathrm{MnP}$ binary bulk compounds is about $291 \mathrm{~K}$ [15]. A magnetic phase diagram for solid solutions of MnP - MnAs was reported in 1968 [16], and magnetic and structural properties of the $\operatorname{MnAs}_{1-y} \mathrm{P}_{y}$ material system have been investigated, mainly for compounds with arsenic rich solid composition such as $y<0.20$. In [16], it is observed that $\mathrm{T}_{\mathrm{c}}$ of $\mathrm{MnAs}_{1-\mathrm{y}} \mathrm{P}_{y}$ changes from about 292 $\mathrm{K}$ to $312 \mathrm{~K}$ with decreasing phosphorus content, $y$, in $\operatorname{MnAs}_{1-y} \mathrm{P}_{y}$, from 1.0 to 0.90 . Therefore, the 
experimental results in the present study presumably indicated that the ferromagnetic coupling of $\mathrm{MnP}$ nanoclusters above room temperature $(305 \mathrm{~K})$ was attributable to the incorporation of about $7 \%$ arsenic atoms into the $\mathrm{MnP}$ clusters, although $\mathrm{T}_{\mathrm{c}}$ for $\mathrm{MnP}$ nanoclusters with $7 \%$ arsenic atoms, which are embedded in InP and/or GaInAs layers, has not been determined precisely yet in this study.

The present results also indicated that there were several differences in the structural and magnetic characteristics of TYPE-I and TYPE-II layer structures between this study and the previous studies [8]. For the TYPE-I layer structures grown by MOVPE using TBAs in [8], (001) planes of hexagonal MnAs nanoclusters were oriented parallel to (111) planes of surrounding GaInAs layers [17]; on the other hand, for the TYPE-I layer structures in this study, which were grown by MOVPE using $\mathrm{AsH}_{3}$, (101) planes of MnAs nanoclusters were oriented nearly parallel to (111) planes of surrounding GaInAs layers. For the TYPE-II layer structures, the ferromagnetic coupling observed in this study was much larger than that in the previous studies [8] when we applied magnetic fields, $\mathbf{H}$, in a direction parallel to the [011] directions. TYPE-I layer structures in the previous study showed much larger ferromagnetic coupling than those in this study. Change in solid compositions of the nanoclusters is possible as well as change in the growth mode of the nanoclusters because we use different precursors such as TBAs in [8] and $\mathrm{AsH}_{3}$ in this study as a group $\mathrm{V}$ source material. We speculate that the surface diffusion of adatoms during the growth of nanoclusters is different when we use different group $\mathrm{V}$ precursors because the pyrolysis processes of TBAs and $\mathrm{AsH}_{3}$ are different, both in the gas phase and on the layer surfaces, and because different optimum V/III ratios in the vapour phase are chosen for the growth using each of the precursors [18]. Detailed angle resolved SQUID measurements, MOVPE growth experiments, electron beam diffraction measurements and EDS analysis are planned to clarify the anisotropy of the magnetic properties with respect to the crystallographic orientations and the solid compositions of the nanoclusters observed here and in the previous study.

\section{Ferromagnetic nanoclusters in Mn-incorporated GaAs layers}

For comparison with the structural and magnetic properties of the Mn-incorporated GaInAs layers, we grew $\mathrm{Mn}$-incorporated 'GaAs' layers by MOVPE using $\mathrm{AsH}_{3}$ as a group V source material. MnAs nanoclusters were also formed in Mn-incorporated GaAs layers grown even at relatively low growth temperatures of $450{ }^{\circ} \mathrm{C}$ as well as ferromagnetic nanocluster formation occurring in the Mn-incorporated GaInAs layers. Figure 6(a) shows cross-sectional TEM images of the Mn-incorporated GaAs layers. Several nanoclusters embedded in GaAs layers were observed (indicated by small white arrows in the top image). The typical nanoclusters measured $80-110 \mathrm{~nm}$ wide and $130-160 \mathrm{~nm}$ high. Figure 6(b) shows a highly magnified lattice image of the nanoclusters. The white rectangular marker in figure 6(a) is a typical observation area for a highly magnified lattice image. As shown in electron beam diffraction patterns in figure 6(b), these nanoclusters had NiAs-type hexagonal crystallographic structures $($ MnAs: $a=0.372 \mathrm{~nm}, \mathrm{c}=0.571 \mathrm{~nm}$ ); on the other hand, the surrounding host matrices had zinc-blende-type crystallographic structures (GaAs). Solid compositions of the observed nanoclusters analyzed by EDS are summarized in the bottom columns in Table 1. The NiAs-type hexagonal nanoclusters embedded in the GaAs layers consisted of a mixture of manganese and arsenic atoms in a solid composition. However, about $4 \%$ gallium atoms were also contained in the nanoclusters. Mn concentrations in the surrounding GaAs layers were less than $1 \%$.

Next, using SQUID magnetometers, we performed magnetic characterizations for the Mn-incorporated GaAs layers. Figure 6(c) shows the growth temperature, $\mathrm{T}_{\mathrm{g}}$, dependence of the M-H curves for Mn-incorporated GaAs layers with MnAs nanoclusters and a reference sample of a GaAs (100) substrate. In all of the measurements, magnetic fields were also applied in a direction parallel to the [011] direction, and the temperature for the SQUID measurements was $10 \mathrm{~K}$. The growth temperatures of the samples were changed from 320 to $450{ }^{\circ} \mathrm{C}$. The results indicate that the lower the growth temperatures the samples were grown at, the larger the saturation magnetization values were. For the growth of the $\mathrm{Mn}$-incorporated GaAs layers at $320,350,370$ and $400{ }^{\circ} \mathrm{C}$, the growth times were the same. Since the growth rate of the epitaxial layers decreases with decreasing growth temperature, the Mn-incorporated GaAs layers grown at $400{ }^{\circ} \mathrm{C}$ were the thickest among these four samples. This is because MOVPE growth processes are limited by the thermochemical reaction kinetics of organometallic source materials at low temperatures below approximately $550{ }^{\circ} \mathrm{C}$ [18]. The growth time for the Mn-incorporated GaAs layers grown at $450{ }^{\circ} \mathrm{C}$ was about half that for the other four samples grown below $400{ }^{\circ} \mathrm{C}$. Therefore, the results shown in figure 6(c) indicated that the larger saturation magnetization tended to be observed in the Mn-incorporated GaAs layers grown at lower growth temperatures, while the thickness of the $\mathrm{Mn}$-incorporated GaAs layers decreased with lowering the growth temperature. The measurements on the 
samples grown at 320,350 and $400{ }^{\circ} \mathrm{C}$ were also made at the relatively high temperature of $270 \mathrm{~K}$. All of these three samples retained clear hysteresis loops up to $270 \mathrm{~K}$ (not shown here). Therefore, even at very low growth temperatures below $400{ }^{\circ} \mathrm{C}$ in the growth by MOVPE, ferromagnetic nanoclusters such as MnAs and/or GaMn were possibly formed in GaAs layers, because the Curie temperature, $T_{c}$, of the MnAs bulk materials is about $313 \mathrm{~K}$, and because it has been reported that the Curie temperatures of the GaMnAs alloy layers and GaMn precipitates were about $110 \mathrm{~K}$ [2] and above $400 \mathrm{~K}$ [4], respectively. The present results on the magnetic characteristics for the samples at various growth temperatures were consistent with the results for the TYPE-II cases shown in the previous sections - however, not with those for the TYPE-I layer structures. Little is known about the formation of ferromagnetic nanoclusters in Mn-incorporated GaInAs layers at present, while GaInMnAs DMS alloys have been investigated [11, 12]. In the TYPE-II layer structures in the present study, we expect MnP nanoclusters to be more easily formed than the MnAs ones because $\Delta \mathrm{H}_{\mathrm{f}}$ for MnAs binary compounds is much larger than that for the MnP ones [13]. For TYPE-I layer structures, XRD measurements indicated that indium contents in Mn-incorporated GaInAs layers were about $4 \%$ lower than those in separate undoped GaInAs reference samples, although we grew TYPE-I layer structures under the same partial pressure conditions of TEGa and TMIn, that is, $\mathrm{p}$ [TEGa] and p[TMIn], as for the undoped GaInAs reference samples. Even in MnP nanoclusters embedded in InP layers in TYPE-II layer structures, the indium contents in the clusters were almost negligible while about $4 \%$ gallium atoms were contained in the MnAs nanoclusters, as indicated in Table 1. Therefore, we speculate that MnAs nanoclusters are more easily formed in Mn-incorporated GaAs layers than in Mn-incorporated GaInAs layers at relatively low growth temperatures about $400{ }^{\circ} \mathrm{C}$ or less.

The size and density of the clusters in the heterostructures become smaller and larger, respectively, with decreasing growth temperature on the basis of the kinetics. This is because the thermally activated surface diffusion of ad-atoms increases with increasing growth temperature. Therefore, smaller MnAs nanoclusters were presumably formed in the $\mathrm{Mn}$-incorporated $\mathrm{GaAs}$ layers grown at lower temperatures, and the densities of the clusters were increased as the growth temperature was lowered from 450 to $320{ }^{\circ} \mathrm{C}$ at the same time. In addition, since a smaller amount of ad-atom re-evaporation occurs with decreasing growth temperature, the total amount of ferromagnetic nanoclusters was presumably increased in the layers grown at lower growth temperatures. The saturation magnetization values, $\mathbf{M}_{\mathbf{s}}$, in figure 6(c) were not divided by the volume or the weight of the samples. However, it may be speculated that, due to the larger amount of smaller MnAs nanoclusters in the Mn-incorporated GaAs layers grown at lower temperatures, a larger saturation magnetization is observed in the samples grown at lower temperatures. It is also likely that the solid composition and/or the crystallographic structure of the nanoclusters themselves, e.g. MnAs, $\mathrm{GaMn}$ and so on, are changed at different growth temperatures of the $\mathrm{Mn}$-incorporated GaAs layers. Detailed cross-sectional TEM observations in combination with EDS analysis of solid compositions in the nanoclusters are under way to clarify the structural characteristics in the Mn-incorporated GaAs layers grown at low growth temperatures below $400{ }^{\circ} \mathrm{C}$.

\section{Conclusions}

In summary, ferromagnetic nanoclusters, which are hybridized in Mn-incorporated GaInAs layers during MOVPE growth on InP (100) layers under low growth temperature conditions below $450{ }^{\circ} \mathrm{C}$, have been demonstrated in this paper. The main results are listed below.

(1) Cross-sectional TEM observations and electron beam diffraction patterns indicated that MnAs nanoclusters with NiAs-type hexagonal crystallographic structures were formed near the layer surfaces of $\mathrm{Mn}$-incorporated GaInAs layers grown at $440{ }^{\circ} \mathrm{C}$. (101) planes of MnAs nanoclusters were oriented nearly parallel to (111) planes of surrounding GaInAs layers. The nanoclusters showed ferromagnetic coupling up to a relatively high temperature of about $305 \mathrm{~K}$.

(2) After deposition of undoped InP layers on Mn-incorporated GaInAs layers, MnP nanoclusters with MnP-type cubic orthorhombic crystallographic structures, in which $7 \%$ arsenic was incorporated, were formed in InP layers. These MnP nanoclusters also showed relatively strong ferromagnetic characteristics up to about $305 \mathrm{~K}$, although the Curie temperature of MnP bulk compounds is $291 \mathrm{~K}$. On the other hand, EDS-analysis showed Mn concentrations in InP and GaInAs layers surrounding the MnP nanoclusters as almost negligible.

(3) We also indicated that ferromagnetic MnAs nanoclusters, which have NiAs-type hexagonal crystallographic structures, were formed in Mn-incorporated GaAs layers grown by MOVPE under low growth temperature conditions: $450{ }^{\circ} \mathrm{C}$ on GaAs (100) substrates. 
These results indicate that ferromagnetic nanoclusters with $T_{c}$ above room temperature were successfully formed in InP and GaInAs layers on InP substrates. We believe that our MOVPE growth technology is promising for hybridizing ferromagnetic nanoclusters into quaternary semiconductor alloys such as GaInAsP and AlGaInAs, preferentially used for optical communication systems.

\section{Acknowledgements}

The authors would like to thank Y. Kotaki for fruitful discussions and continuous encouragement. The authors are also grateful to A. Akasegawa and K. Yamanaka for helpful suggestions and fruitful discussions regarding SQUID measurements, and Dr. P. Mohan of Hokkaido University for a critical reading of the paper. Finally, the authors would like to express their sincere appreciation to Dr. W. Stolz of Philipps-University Marburg and Professor T. Fukui of Hokkaido University for stimulating discussions and fruitful suggestions. 


\section{References}

[1] Tanaka M 1999 J. Cryst. Growth 201/202 660 for a review, and see reference papers therein.

[2] Ohno H 1999 J. Magn. Magn. Mater. 200110 for a review, and see reference papers therein.

[3] Munekata H, Ohno H, von Molnar S, Segmüller A, Chang L L and Esaki L 1989 Phys. Rev. Lett. 63 1849; Koshihara S, Oiwa A, Hirasawa M, Katsumoto S, Iye Y, Urano C, Takagi H and Munekata H 1997 Phys. Rev. Lett. 784617

[4] Shi J, Kikkawa J M, Awschalom D D, Medeiros-Ribeiro G, Petroff P M and Babcock K 1996 J. Appl. Phys. 795296

[5] Hartmann Th, Lampalzer M, Stolz W, Megges K, Lorberth J, Klar P J and Heimbrodt W 2000 Thin Solid Films 364209

[6] Heimbrodt W, Hartmann Th, Klar P J, Lampalzer M, Stolz W, Volz K, Schaper A, Treutmann W, Krug von Nidda H - A, Loidl A, Ruf T and Sapega V F 2001 Physica E 10175

[7] Blattner A J and Wessels B W 2002 J. Vac. Sci. Technol. B 201582

[8] Hara S, Lampalzer M, Torunski T, Volz K, Treutmann W and Stolz W 2004 J. Cryst. Growth 261330

[9] Yokoi H, Mizumoto T, Shimizu M, Futakuchi N, Kaida N and Nakano Y 1999 Japan. J. Appl. Phys. 38 195

[10] Shimizu H and Tanaka M 2002 Appl. Phys. Lett. 815246

[11] Slupinski T, Munekata H and Oiwa A 2002 Appl. Phys. Lett. 80 1592; Slupinski T, Munekata H and Oiwa A 2002 J. Cryst. Growth 237 - 2391331

[12] Ohya S, Shimizu H, Higo Y, Sun J and Tanaka M 2002 Japan. J. Appl. Phys. 41 L24

[13] Barin I 1989 Thermochemical Data of Pure Substances (Weinheim: VCH)

[14] Malik S K and Vijayaraghavan R 1969 Rhys. Lett. A 28648

[15] Huber E E Jr and Ridgley D H 1964 Phys. Rev. 135 A1033

[16] Roger A and Fruchart R 1968 Mater. Res. Bull. 3253

[17] Leuschner M, Klar P J, Heimbrodt W, Rühle W W, Hara S, Stolz W, Volz K, Kurz T, Loidl A and Krug von Nidda H - A J. Magn. Magn. Mater. in press

[18] Stringfellow G B 1999 Organometallic Vapor-Phase Epitaxy: Theory and Practice (San Diego, CA: Academic) 
Figure 1 Supply system set-up for $(\mathrm{MeCp})_{2} \mathrm{Mn}$ in the MOVPE system. In order to introduce manganese precursors efficiently to the wafer surfaces, the temperatures of $(\mathrm{MeCp})_{2} \mathrm{Mn}$ sources and their gas inlets to the reactor were heated to 70 and $75^{\circ} \mathrm{C}$, respectively.

Figure 2 Cartoon of typical layer structures for Mn-incorporated GaInAs layers. In order to study growth morphologies and magnetic properties of magnetic nanoclusters in heterostructures, 'TYPE-I' (without undoped InP cap layers) and 'TYPE-II' (with undoped InP cap layers) layer sequences were chosen in this study.

Figure 3 Cross-sectional TEM images and electron beam diffraction patterns for (a) TYPE-I and (b) TYPE-II layer sequences. Highly magnified lattice images of nanoclusters and surrounding layers were also observed. The white rectangular marker in the TEM image of (b) is a typical observation area for taking a highly magnified image. TEM images were taken with [011] projection for surrounding zinc-blende GaInAs layers in (a), and taken with [0-11] projection for surrounding zinc-blende GaInAs and InP layers in (b). In the TYPE-II layer sequence in (b), a 'trilayer' heterostructure sample was grown only for cross-sectional TEM observations, as depicted in the schematic illustration. Designed layer thicknesses of the samples were as follows: undoped InP cap and spacer layers $(80 \mathrm{~nm}) / \mathrm{Mn}$-incorporated GaInAs (150, 120 and $100 \mathrm{~nm}$ ). Nanoclusters formed in the layers are indicated by white arrows in the images.

Figure 4 SQUID measurement temperature dependences of M-H curves for the TYPE-I layer sequence grown at (a) $440{ }^{\circ} \mathrm{C}$ and (b) $380{ }^{\circ} \mathrm{C}$. All data were corrected for the diamagnetism of undoped GaInAs/InP reference samples.

Figure $5 \mathrm{M}-\mathrm{H}$ curves for the TYPE-II and an undoped GaInAs/InP reference sample. Dependence on (a) growth temperatures, $\mathrm{T}_{\mathrm{g}}$, of the samples, (b) $\mathrm{Mn} /(\mathrm{III}+\mathrm{Mn})$ ratios in the vapour phase, $x$, for the Mn-incorporated GaInAs layers and (c) SQUID measurement temperatures. Data were not corrected for the diamagnetism of undoped GaInAs/InP reference samples.

Figure 6 Ferromagnetic nanocluster formation in reference samples of Mn-incorporated GaAs layers. (a) Cross-sectional TEM image of nanoclusters. The growth temperature was $450{ }^{\circ} \mathrm{C}$. (b) Highly magnified lattice images and electron beam diffraction patterns of a MnAs nanocluster and surrounding GaAs layers. TEM images were taken with [0-11] projection for surrounding zinc-blende GaAs layers. (c) $\mathrm{M}-\mathrm{H}$ characterization results of the samples as a function of growth temperatures, $T_{g}$, for MOVPE growth. Data were not corrected for the diamagnetism of undoped GaAs reference samples. 
Table 1 Averaged atomic concentration (at. \%) of five elements, In, Ga, As, P and Mn, in GaInAs layers, InP layers and nanoclusters, respectively, analyzed using EDS for both TYPE-I and TYPE-II layer structures. The focused spot diameter of the electron beam for the EDS analysis was about $1 \mathrm{~nm}$. The deviation of the average values shown here is around \pm 2 (at. \%) at most. As a reference, EDS analysis results for the samples of Mn-incorporated GaAs layers grown on GaAs substrates are also summarized in the bottom columns.

\begin{tabular}{cccccc}
\hline \hline \multirow{2}{*}{$\begin{array}{c}5 \\
\text { Lamples) }\end{array}$} & \multicolumn{5}{c}{ Averaged atomic concentration in layers (\%) } \\
\cline { 2 - 6 } (TYPE-I) & In & Ga & As & P & Mn \\
GaInAs & $\mathbf{2 2 . 0}$ & $\mathbf{2 7 . 8}$ & $\mathbf{4 8 . 4}$ & 1.5 & 0.3 \\
Clusters & 2.6 & 0.3 & $\mathbf{5 4 . 3}$ & 1.8 & $\mathbf{4 1 . 0}$ \\
\hline (TYPE-II) & & & & \\
GaInAs & $\mathbf{2 4 . 0}$ & $\mathbf{2 5 . 5}$ & $\mathbf{4 9 . 5}$ & 0.7 & 0.3 \\
InP & $\mathbf{5 0 . 4}$ & 0.2 & 2.1 & $\mathbf{4 7 . 0}$ & 0.3 \\
Clusters & 0.4 & 0.2 & $\mathbf{7 . 2}$ & $\mathbf{4 8 . 5}$ & $\mathbf{4 3 . 7}$ \\
\hline (Reference) & & & & & \\
GaAs & - & $\mathbf{4 7 . 9}$ & $\mathbf{5 1 . 5}$ & - & 0.4 \\
Clusters & - & $\mathbf{3 . 8}$ & $\mathbf{5 3 . 7}$ & - & $\mathbf{4 2 . 3}$ \\
\hline \hline
\end{tabular}




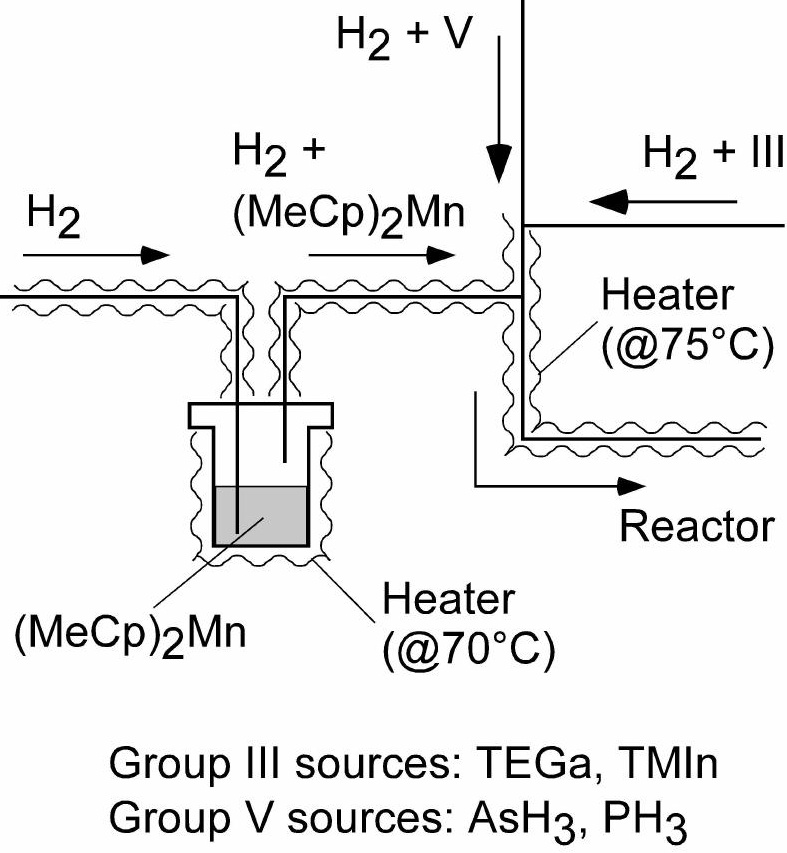


Mn-incorporated GalnAs $(<100 \mathrm{~nm})$

\section{undoped InP cap (10 nm)}

undoped GalnAs $(100 \mathrm{~nm})$
Mn-incorporated GalnAs $(<100 \mathrm{~nm})$ undoped InP buffer $(100 \mathrm{~nm})$ $\mathrm{n}^{+}$-type $\ln \mathrm{P}(100)$ substrate undoped InP buffer $(100 \mathrm{~nm})$

$\mathrm{n}^{+}$-type $\ln \mathrm{P}(100)$ substrate
TYPE-I 

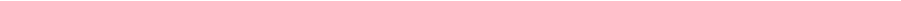

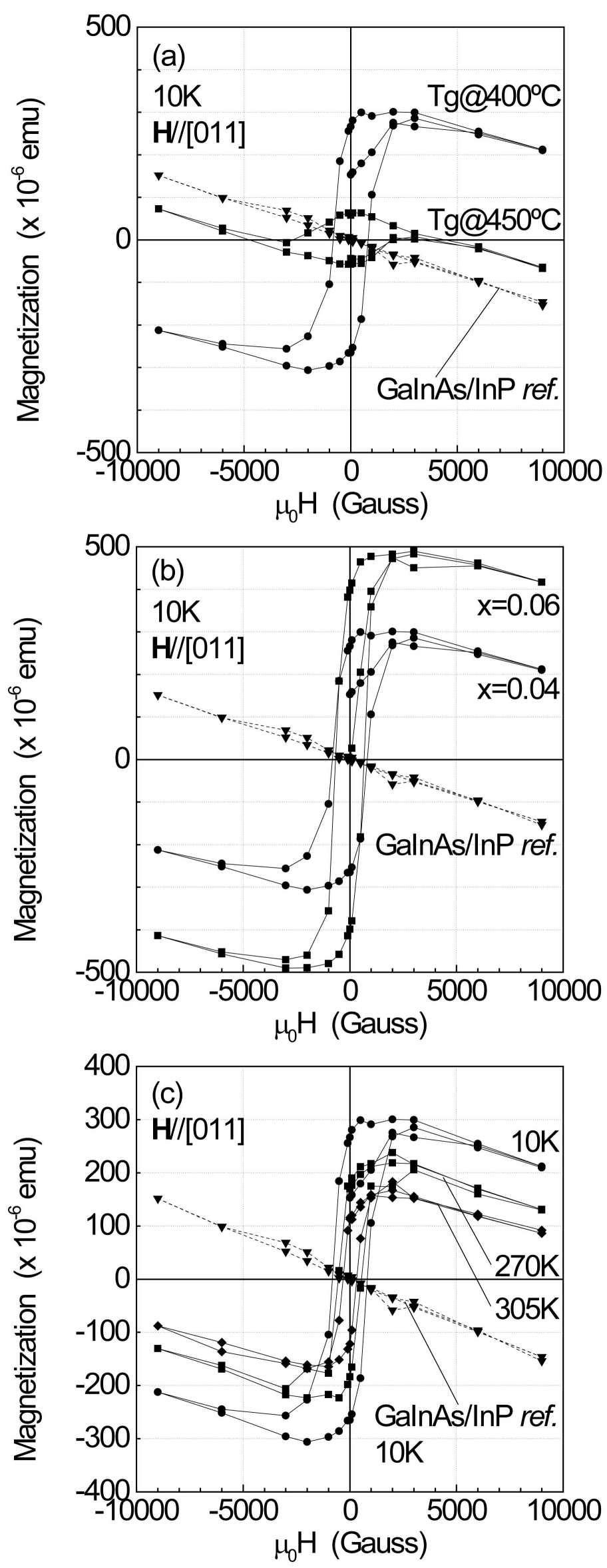
